\title{
MUERTE MATERNA EN CHILE POR ECLAMPSIA
}

En el mundo aproximadamente fallecen algo más de 500.000 mujeres producto de causas relacionadas con el embarazo, parto o puerperio (TMM promedio en 2005 de 402/100.000 NV), de ellas 15.500 ocurren en América Latina y el Caribe (TMM promedio en 2005 de 132/100.000 NV) (1).

La muerte materna asociada al síndrome hipertensivo del embarazo en muchos países es la principal causa de muerte. En América Latina y el Caribe es la primera causa de muerte materna con una frecuencia promedio de $25,7 \%$ (2). En el embarazo, el $10 \%$ de las mujeres presentará hipertensión arterial y la preeclampsia complicará entre el 2 - 8\% de los embarazos; esta última es una enfermedad multisistémica, siendo su expresión máxima el síndrome de HELLP y la eclampsia (3). La muerte por eclampsia en Europa es de baja frecuencia, con 2-3 casos por 10.000 partos; mientras que en países en desarrollo se estima una incidencia de 16 a 69 casos por 10.000 partos (3). En los países con tasas altas de mortalidad materna, la muerte por eclampsia es superior a la por preeclampsia, revirtiéndose esta proporción en la medida que desciende la mortalidad materna global (4).

Es importante conocer en forma global y preliminar lo que acontece en nuestro país. En Chile, en el período 1990-2000, la hipertensión arterial fue la primera causa de mortalidad materna, con 181 fallecimientos y una TMM promedio de 5,8/100.000 $\mathrm{NV}$, siendo una de las 3 causas que presentaron un descenso significativo y estrechamente relacionadas con el descenso de la natalidad (5). Antes de 1997 desconocemos las muertes por eclampsia, debido a que en el país las estadísticas vitales se registraban según la novena revisión de la Clasificación Internacional de Enfermedades, Traumatismos y Causas de Defunción (CIE-9), a partir de 1997 se clasifican según la décima revisión (CIE10 ), que considera la muerte materna por eclampsia bajo el código 015.

En el período 1997-2007, en Chile hubo 70 muertes maternas por eclampsia y 2.699.285 na- cidos vivos corregidos, con una TMM promedio de 2,6/100.000 NV (Tabla I). Se aprecia una tendencia descendente y significativa de la natalidad $(r=-0,8871 ; p<0,001)$, de la tasa de mortalidad por eclampsia $(r=-0,7142 ; p<0,01)$ y de la tasa de natalidad $(r=-0,9559 ; p<0,001)$, como también una asociación directa y significativa entre la tasa de mortalidad materna por eclampsia y la tasa de natalidad $(r=+0,6844$; $p<0,02)$; pero sin cambios significativos desde el 2003 en adelante. En la Figura 1 se grafica la tendencia de la TMM por eclampsia.

Los datos presentados son promisorios, sin embargo, pueden ser mejores, estamos aún lejos de países desarrollados de la región de las Américas y de Europa. Es así que en EEUU en 2006 la TMM por preeclampsia/eclampsia fue de 1,3/100.000 NV (6), en Canadá en 2008 para síndrome hipertensivo fue de 0,6/100.000 NV (7) y en el Reino Unido en el período 2003-2005 para preeclampsia/eclampsia fue de 0,85/100.000 NV (8).

La tendencia de las cifras presentadas debemos mantenerlas e idealmente disminuirlas. Chile posee una amplia cobertura del control prenatal y de la atención institucional/profesional del parto, que en nuestro país supera el 90 y $99 \%$ respectivamente; lo anterior junto al control de la fecundidad son las estrategias más importantes para controlar la muerte materna asociadas a hipertensión arterial, como también a todas las causas en general y muy especialmente en la población de alto riesgo obstétrico (9).

Debemos estar alertas a los cambios epidemiológicos y demográficos del país que muestran el envejecimiento poblacional de la mujer chilena en edad fértil (10) y el aumento de la natalidad en adolescentes (11) y en edad materna avanzada (12), ambos grupos de alto riesgo de síndrome hipertensivo y de eventual mortalidad materna.

Dr. Enrique Donoso S. Editor Jefe. 
Tabla I

NÚMERO DE MUERTES MATERNAS POR ECLAMPSIA, NACIDOS VIVOS CORREGIDOS, TASA DE MORTALIDAD MATERNA POR ECLAMPSIA Y TASA DE NATALIDAD, CHILE 1997-2007

\begin{tabular}{ccccc}
\hline Año & $\begin{array}{c}\text { Muertes por } \\
\text { eclampsia }\end{array}$ & $\begin{array}{c}\text { Recién nacidos } \\
\text { corregidos }\end{array}$ & $\begin{array}{c}\text { TMM por } \\
\text { eclampsia * }\end{array}$ & $\begin{array}{c}\text { Tasa de } \\
\text { natalidad }^{* *}\end{array}$ \\
\hline 1997 & 7 & 265.493 & 2,6 & 17,9 \\
1998 & 8 & 261.802 & 3,1 & 17,5 \\
1999 & 11 & 254.096 & 4,3 & 16,7 \\
2000 & 9 & 252.155 & 3,6 & 16,4 \\
2001 & 10 & 248.651 & 4,0 & 16,0 \\
2002 & 8 & 241.027 & 3,3 & 15,3 \\
2003 & 3 & 236.223 & 1,3 & 14,8 \\
2004 & 4 & 232.588 & 1,7 & 14,5 \\
2005 & 3 & 232.092 & 1,3 & 14,3 \\
2006 & 3 & 233.104 & 1,3 & 14,2 \\
2007 & 4 & 242.054 & 1,7 & 14,6 \\
\hline
\end{tabular}

*Tasa por 100.000 nacidos vivos. ${ }^{* *}$ Tasa por 1000 habitantes. Fuente: Instituto Nacional de Estadísticas.

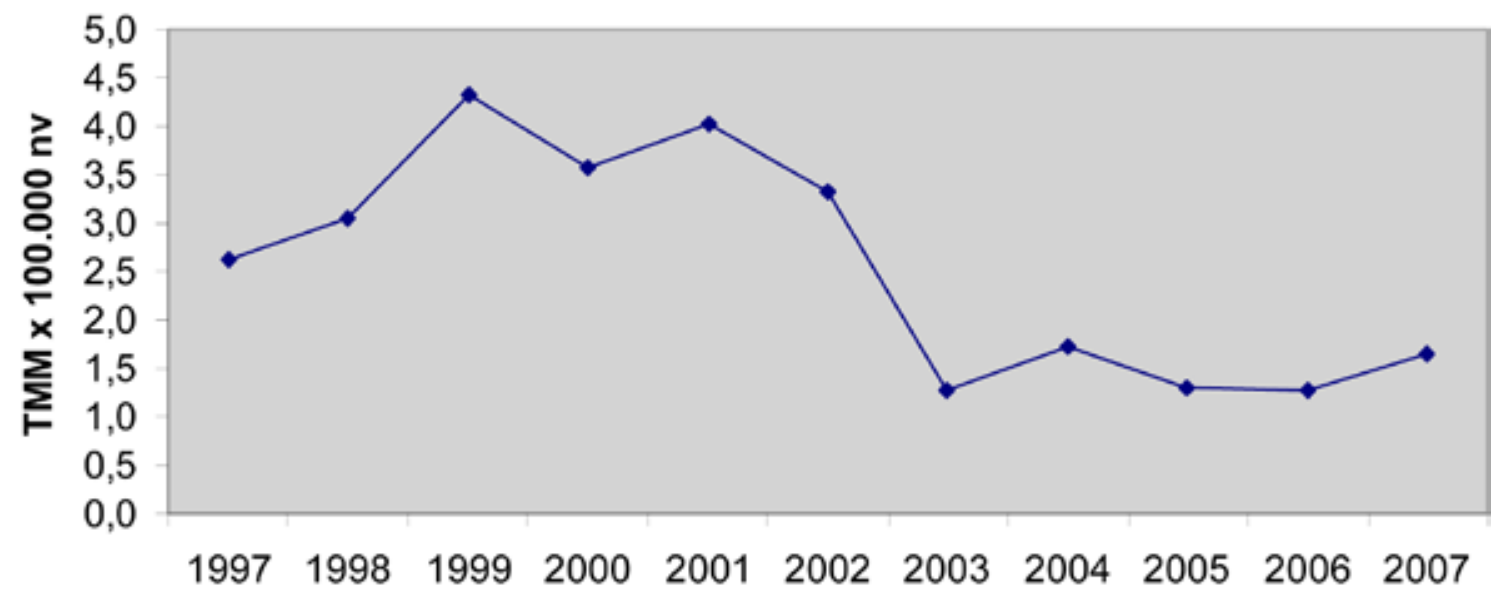

Figura 1. Tendencia de la tasa de mortalidad materna por eclampsia, Chile 1997-2007.

\section{REFERENCIAS}

1. Hill K, Thomas K, AbouZahr C, Walker N, Say L, Inoue M, Suzuki E; Maternal Mortality Working Group. Estimates of maternal mortality worldwide between 1990 and 2005: an assessment of available data. Lancet 2007;370(9595):1311-9.

2. Khan KS, Wojdyla D, Say L, Gülmezoglu AM, Van Look PF. WHO analysis of causes of maternal death: a systematic review. Lancet 2006;367(9516):1066-74.

3. Duley $\mathrm{L}$. The global impact of pre-eclampsia and eclampsia. Semin Perinatol 2009;33(3):130-7.

4. Duley L. Maternal mortality associated with hypertensive disorders of pregnancy in Africa, Asia, Latin America and the Caribbean. Br J Obstet Gynaecol. 1992
Jul;99(7):547-53.

5. Donoso E. [The reduction in maternal mortality in Chile, 1990-2000]. Rev Panam Salud Publica 2004;15(5):326-30. [Article in Spanish].

6. Heron M, Hoyert DL, Murphy SL, Xu J, Kochanek KD, Tejada-Vera B. Deaths: final data for 2006. Natl Vital Stat Rep 2009;57(14):1-134.

7. Bartholomew S, Liston R, O'Brien B. Maternal health outcomes. 15. Maternal mortality ratio. En: Public Health Agency of Canada. Canadian Perinatal Health Report, 2008 Edition. Ottawa, 2008.

8. Neilson J. 3. Pre-eclampsia and eclampsia. En: The confidential enquiry into maternal and child health (CEMACH). Saving mother's live: reviewing maternal deaths to make motherhood safer 2003-2005. The 
seventh report on confidential enquiries into maternal deaths in the United Kingdom. London: CEMACH.

9. Stover J, Ross J. How increased contraceptive use has reduced maternal mortality. Matern Child Health $\mathrm{J}$ 2010;14(5):687-95.

10. Donoso E, Carvajal J, Domínguez MA. [Fecundity reduction and aging in fertile women population in the period 1990-2004 in Chile]. Rev Med Chil 2009;137(6):766-73. [Article in Spanish]
11. Donoso E, Becker J, Villarroel L. [Birth rates and reproductive risk in adolescents in Chile, 1990-1999]. Rev Panam Salud Publica 2003;14(1):3-8. [Article in Spanish]

12. Donoso E, Becker J, Villarroel L. Evolución de la natalidad y del riesgo reproductivo en mujeres de 40 o más años en la década de los 90. Rev Chil Obstet Ginecol 2002;67(2):139-42. 